\title{
Breast Cancer Education
}

National Cancer Institute

\section{Source}

National Cancer Institute. Breast Cancer Education. NCI Thesaurus. Code C18662.

Programs or materials to provide information on breast cancer prevention, detection, or treatment to health professionals, patients and their families, the public, or high-risk groups. 